\title{
Tabletop EUV Coherent Diffractive Imaging and Small Angle Scattering of Colloidal Crystals
}

\author{
Giulia F. Mancini ${ }^{1,2,3}$, Robert Karl Jr. ${ }^{1}$, Elisabeth Shanblatt ${ }^{1}$, Charles Bevis ${ }^{1}$, Dennis Gardner ${ }^{1}$, \\ Michael Tanksalvala ${ }^{1}$, Jennifer Russell ${ }^{4,6}$, Daniel Adams ${ }^{1}$, Henry Kapteyn ${ }^{1,7}$, \\ John Badding ${ }^{4,5,6}$, Thomas Mallouk ${ }^{4,5,6}$ Margaret Murnane ${ }^{1,7}$ \\ ${ }^{1}$ JILA, 440 UCB, University of Colorado, Boulder, CO 80309 USA \\ ${ }^{2}$ Laboratory for Ultrafast Spectroscopy, Lausanne Center for Ultrafast Science (LACUS), École Polytechnique Fédérale de Lausanne, CH- \\ 1015 Lausanne, Switzerland. ${ }^{3}$ Paul Scherrer Institut, WSLA/210, 5232 PSI Villigen, Switzerland. \\ ${ }^{4}$ Department of Chemistry, Penn State University, University Park, PA 16802, United States. \\ ${ }^{5}$ Department of Physics, Penn State University, University Park, PA 16802, United States. \\ ${ }^{6}$ Materials Research Institute, Penn State University, University Park, PA 16802, United States. \\ ${ }^{7}$ KMLabs Inc., 4775 Walnut Street, Suite 102, Boulder, CO 80301, United States. \\ E-mail: giulia.mancini@colorado.edu
}

\begin{abstract}
We demonstrate full-field quantitative ptychographic imaging using tabletop high harmonics to visualize the extended structure of silica close-packed nanosphere multilayers with $<20 \mathrm{~nm}$ spatial resolution, and also extract small-angle EUV Bragg scattering order/disorder correlations.
\end{abstract}

\section{Introduction}

Quantitative, non-destructive full-field imaging is an essential tool for understanding nanoscale systems. In particular, tabletop extreme ultraviolet (EUV) coherent diffractive imaging (CDI) techniques based on high harmonic generation $(\mathrm{HHG})$ are ideal for investigating complex nanostructured systems, as they combine elemental and chemical selectivity with nanometer spatial resolution, with pulse durations in the femtosecond (fs)-to-attosecond (as) range[1,2]. In recent work, we demonstrated the first sub-wavelength spatial resolution full field imaging using any short wavelength light source, small or large[3]. Moreover, with recent advances in algorithms, we demonstrated that quantitative full-field imaging is now possible with EUV CDI desktop microscopes, enabling a unique new capability for materials and nano science.

In this work, we apply full-field, quantitative ptychographic HHG CDI[4,5] to visualize the extended structure of a silica periodic close-packed nanosphere multilayer, with a spatial resolution better than $20 \mathrm{~nm}$. In our images, thickness information is encoded in the phase and the spatially dependent transmissivity is contained in the amplitude. We also demonstrate small-angle Bragg scattering from these nanostructured materials, to reveal information about the local order of nanosphere grains, separated by grain boundaries and discontinuities.

\section{Experimental Methods}

We used a $13 \mathrm{~nm}$ high harmonic source (KMLabs XUUS 4.0) driven by a $20 \mathrm{fs}, 2 \mathrm{~mJ}, 3 \mathrm{kHz}$, Ti:Sapphire laser centered at $785 \mathrm{~nm}$ (KMLabs Dragon) to generate ultrastable HHG beams as the illumination of our ptychographic microscope. Bright, phase matched, spatially coherent HHG beams at a wavelength of $13.5 \mathrm{~nm}$ were produced in a $150 \mu \mathrm{m}$ diameter waveguide filled with He at 575 Torr. The residual laser light was rejected using a pair of $\mathrm{ZrO}_{2}$ coated Si mirrors placed near Brewster's angle, and a $400 \mathrm{~nm} \mathrm{Zr}$ foil. A single harmonic at a wavelength of $13.5 \mathrm{~nm}$ was then selected using a pair of $\mathrm{Si} / \mathrm{Mo}$ multilayer mirrors. The first flat mirror is used to steer the beam onto the second curved mirror. The nanosphere quasi-periodic close-packed multilayer was imaged with ptychography CDI. In ptychography, multiple diffraction patterns are collected by scanning the illuminating beam across the sample, such that each scan position overlaps with adjacent positions[1,4,5]. We scanned the sample in the focus of the EUV beam ( $5 \mu \mathrm{m}$ diameter) in a rectilinear pattern, with 81 positions, with a random offset of $\pm 30 \%$ of the step size. The sample was placed $18.3 \mathrm{~mm}$ away from the detector (Fig.1(a.b)). At each scan position, 2 frames were acquired with an exposure time of $4 \mathrm{sec}$, at $1 \mathrm{MHz}$ pixel readout rate. For small-angle scattering experiments the EUV beam focus size was increased to $15 \mu \mathrm{m}$ and the nanosphere monolayer was placed $15.1 \mathrm{~mm}$ away from the detector.

\section{Results and Discussion}

The ptychographic reconstructions are shown in Fig. 1(c,d). The numerical aperture of our diffraction patterns is 0.37 , which supports a resolution $\Delta_{r}=\lambda / 2 N A$ of $18.2 \mathrm{~nm}$. From the reconstructed phase Fig. 1(d,h), we can determine the thickness of silica at each spatial location. Comparing a region at the center of a nanosphere to a region in between nanospheres, we find a phase difference of $0.16 \mathrm{rad}$, which corresponds to a thickness of $124 \mathrm{~nm}$, which is in good agreement with the estimated nanosphere size from the SEM images. The amplitude reconstruction (Fig. 1c) shows quantitatively the spatially dependent transmissivity of the sample. Comparing a high transmission region to a low transmission region, we see that the ratio of the transmissivities is 0.54 , which is in agreement with the transmission through $124 \mathrm{~nm}$ of silica. Furthermore, the values of the transmissivity 

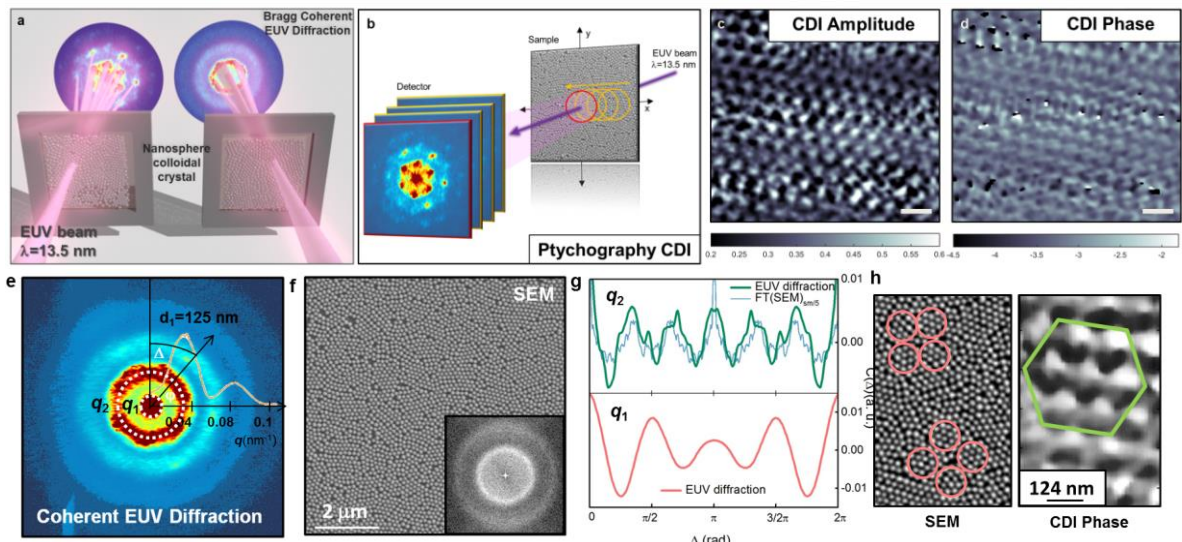

Fig. 1. (a) X-ray scattering and microscopy of silica nanosphere colloidal crystals with different ordered and structure. (b) Schematic of transmission-mode EUV ptychography. (c, d) Reconstructed quantitative amplitude and phase. The scale bars are $250 \mathrm{~nm}$. (e) Coherent diffraction pattern of a silica nanosphere monolayer. (f) SEM image of the sample. Inset: FT simulation of the SEM image. (g) Angular cross-correlation analysis at $q_{2}$ (top) and $q_{1}$ (bottom). (h) Left: visualization of four-fold arrangement of grains of silica nanospheres in the SEM image. Right: the reconstructed phase confirms the hexagonal arrangement of nanospheres within grains in the colloidal crystal and yields at nanosphere thickness of $124 \mathrm{~nm}$.

range from 0.2 to 0.6 , which correspond to transmission through $30 \mathrm{~nm}$ of silicon nitride followed by transmission through $248 \mathrm{~nm}$ and $124 \mathrm{~nm}$ of silica, respectively, indicating that the imaged region is a multilayer. In our experiment, the angular cross-correlation analysis $(\mathrm{CCF})[6,7]$ at small-angle scattering vectors reveals important information on the arrangement of grains containing several nanospheres $\left(q_{1}\right)$, and of the nanospheres therein $\left(q_{2}\right)$ (Fig.1 $(\mathrm{e}, \mathrm{g})$ ). The CCF calculated from the EUV diffraction pattern at $q_{2}=0.05 \mathrm{~nm}^{-1}$ reveals a local hexagonal arrangement of nanospheres within grains, confirmed by the CCF from the FT of the SEM image of the nanosphere monolayer (Fig.1f) and from the reconstructions (Fig,1 (c,d,h)). The inter-particle distance retrieved with diffraction is $125 \pm 2 \mathrm{~nm}$, in agreement with the reconstructed phase image (124nm) and with SEM $(123 \pm 3 \mathrm{~nm})$. The symmetry of a few nearest-neighbor grains of average center-center size $\approx 1 \mu \mathrm{m}$, is obtained calculating the CCF at $q_{1} \approx 0.006 \mathrm{~nm}^{-1}$, and reveals that in this portion of the monolayer, grains that include several nanospheres have an average nearest-neighbour arrangement that is preferentially four-fold (Fig. 1h).

\section{Conclusions}

We demonstrated that EUV coherent diffractive imaging can be used to non-destructively visualize periodically extended self-assemblies of multilayered nanospheres and to retrieve quantitative information about the sample with a spatial resolution better than $20 \mathrm{~nm}$. Moreover, we presented the first demonstration of small-angle Bragg EUV scattering from nanostructures using a tabletop-scale setup, enabling structural characterization of colloidal crystals of different packing symmetry. Harnessing the ultrafast temporal resolution of high harmonic sources $\left(<10^{-15} \mathrm{sec}\right)$ combined with chemical, elemental and spin specificity, means that this approach can be extended to capture 3D structural and dynamic properties[8] of many different types of functional nanomaterials, with broad application in material science and nanotechnology.

\section{Acknowledgements}

The Penn State Center for Nanoscale Science, an NSF-sponsored Materials Science and Engineering Center under award DMR-1420620, DARPA PULSE: W31P4Q-13-1-0015, Gordon and Betty Moore Foundation's EPiQS Initiative through Grant GBMF: 4538, Swiss National Science Foundation (SNSF) grant No.P2ELP2_158887, NDSEG fellowship, NSF GRFP Fellowship.

\section{References}

[1] M. Seaberg, B. Zhang, D. Gardner, E. Shanblatt, M. Murnane, H. Kapteyn, D. Adams, "Tabletop nanometer extreme ultraviolet imaging in an extended reflection mode using coherent Fresnel ptychography" Optica 1, 39-44 (2014).

[2] J. Miao, T. Ishikawa, I. K. Robinson \& M. M. Murnane, "Beyond Crystallography: Diffractive Imaging with Coherent X-ray Sources", Science 348, 530 (2015),

[3] D. Gardner, M. Tanksalvala, E. Shanblatt, X. Zhang, B. Galloway, C. Porter, R. Karl, C. Bevis, D. Adams, H. Kapteyn, M. Murnane, and G. Mancini, "Subwavelength coherent imaging of periodic samples using a $13.5 \mathrm{~nm}$ tabletop high-harmonic light source," Nat. Photonics, 11, 259 (2017).

[4] P. Thibault., M Dierolf, O Bunk, A Menzel, and F Pfeiffer, "Probe retrieval in ptychographic coherent diffractive imaging," Ultramicroscopy, 109, 338 (2009).

[5] M. Maiden and J. Rodenburg, "An improved ptychographical phase retrieval algorithm for diffractive imaging," Ultramicroscopy 1091256 (2009).

[6] M. Altarelli, R. P. Kurta, and I. A.Vartanyants, "X-ray cross-correlation analysis and local symmetries of disordered systems: General theory", Phys. Rev. B 82, 104207 (2010).

[7] G. F. Mancini, T. Latychevskaia, F. Pennacchio, J. Reguera, F. Stellacci, and F. Carbone, "Order/disorder dynamics in a dodecanethiol-capped gold nanoparticles supracrystal by smallangle ultrafast electron diffraction", Nano Lett. 16, 2705-2713 (2016).

[8] R. M. Karl, G. F. Mancini, D. Gardner, E. Shanblatt, C. Bevis, J. Knobloch, T. Frazer, J. N. Hernandez-Charpak1, B. Abad Mayor, E. Shanblatt, M. Tanksalvala, C. Porter, D. Adams, H. Kapteyn and M. M. Murnane, "Ultrafast Dynamic Imaging of Acoustic Waves in Nanosystems with a Tabletop High Harmonic Source," Conference on Lasers and Electro-Optics Postdeadline paper 2017, JTh5C.8. 